\title{
Frontières
}

\section{La mort et le deuil en contexte scolaire}

\section{Michel Hanus}

Volume 13, numéro 1, automne 2000

La mort au tableau noir

URI : https://id.erudit.org/iderudit/1074245ar

DOI : https://doi.org/10.7202/1074245ar

Aller au sommaire du numéro

Éditeur(s)

Université du Québec à Montréal

ISSN

1180-3479 (imprimé)

1916-0976 (numérique)

Découvrir la revue

Citer cet article

Hanus, M. (2000). La mort et le deuil en contexte scolaire. Frontières, 13(1), 36-39. https://doi.org/10.7202/1074245ar

\section{Résumé de l'article}

Une prise de conscience de l'importance de savoir vivre la mort et le deuil tous ensemble dans les établissements d'enseignement est en voie de se réaliser actuellement tant du côté des personnels de ces établissements que de celui des responsables d'académie. Actuellement des formations se mettent en place assez largement à travers la France en vue de réaliser un protocole d'intervention en cas de survenue d'une situation de deuil dans l'établissement, en particulier par suicide. Mais un tel travail nécessite d'acquérir des connaissances sur les conceptions de la mort et le déroulement du deuil chez les enfants et les adolescents.
Ce document est protégé par la loi sur le droit d'auteur. L'utilisation des services d'Érudit (y compris la reproduction) est assujettie à sa politique d'utilisation que vous pouvez consulter en ligne.

https://apropos.erudit.org/fr/usagers/politique-dutilisation/ 


\section{Résumé}

Une prise de conscience de l'importance de savoir vivre la mort et le deuil tous ensemble dans les établissements d'enseignement est en voie de se réaliser actuellement tant du côté des personnels de ces établissements que de celui des responsables d'académie. Actuellement des formations se mettent en place assez largement à travers la France en vue de réaliser un protocole d'intervention en cas de survenue d'une situation de deuil dans l'établissement, en particulier par suicide. Mais un tel travail nécessite d'acquérir des connaissances sur les conceptions de la mort et le déroulement du deuil chez les enfants et les adolescents.

Mots clés : mort-deuil-formation

\section{Abstract}

An awakening of the importance of death and mourning at school is in the process of becoming a reality, both by the principal and teachers and by those who are in charge of curriculum development. At present, people are being trained throughout France in order to intervene in case a student dies particularly by suicide. However such work necessitates the acquisition of knowledge on issues surrounding death and mourning among children and adolescents.

Key words : death-mourning - curriculum development

\section{La mort \\ et le deuil en contexte scolaire}

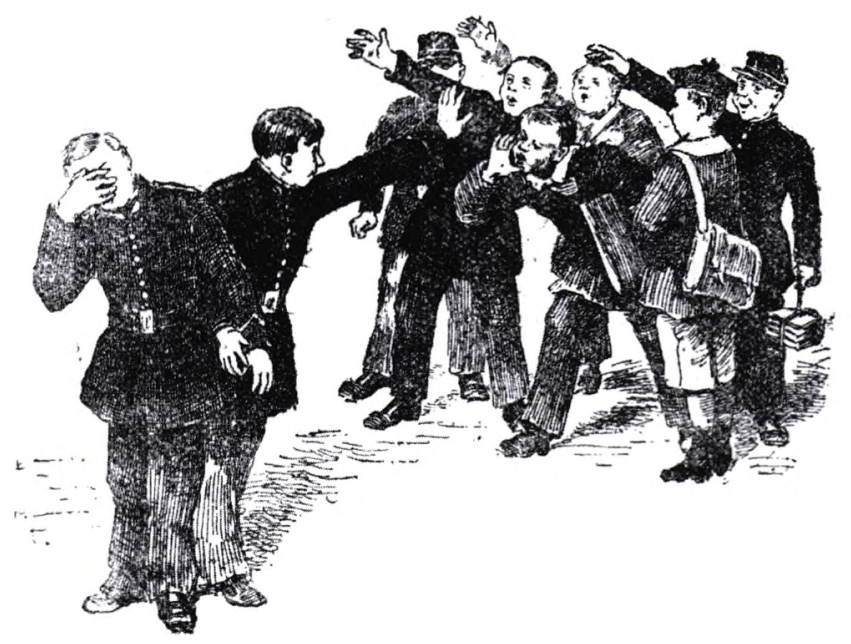

Michel Hanus,

médecin et président de la Société de Thanatologie et de I'association française Vivre son Deuil.

Depuis quelques décennies, la mort a été mise en retrait du monde scolaire. Dans une perspective générale, elle n'était plus abordée que dans l'enseignement de l'histoire, de la littérature et de la biologie. Du temps, plus ancien, où l'instruction civique faisait partie du programme de l'enseignement dans les écoles élémentaires de la France, alors appelées "écoles communales ", la mort était sujet d'actualité au moment de la Toussaint, certains instituteurs emmenaient alors leur classe au cimetière. J'imagine ces gamins devant l'entrée d'un cimetière rural de la Manche où il est écrit "Nous avons été ce que vous êtes, vous serez ce que nous sommes "! Cela fait un beau sujet de réflexion et de discussion dans la classe. La mort, habituellement si lointaine dans la pensée des enfants, devient promptement beaucoup plus réelle.

L'occultation sociale de la mort dans les établissements scolaires ne fait que refléter le même phénomène dans l'ensemble de la société contemporaine.
Mais lorsque survient la mort d'un élève ou d'un adulte de l'établissement, les membres de la direction semblent souvent démunis, déroutés, tiraillés entre le sentiment de l'utilité d'en parler et le désir de ne pas faire de vague. On se conduit comme si la mort ne faisait pas partie des missions éducatives de l'institution.

En réalité, les réactions sont très variables selon les établissements, les circonstances et les responsables scolaires. En présence d'un événement tragique, bien des chefs d'établissement préféreraient qu'on en parle le moins possible, alors que d'autres membres du personnel, en particulier médico-sociaux et éventuellement enseignants, sentent la nécessité d'en parler avec tous les membres de l'établissement qui se trouvent alors en deuil. Ainsi les réactions institutionnelles sont parfois marquées par un silence complet : la vie semble continuer comme s'il ne s'était rien passé alors que des modifications sont perceptibles dans le fonctionnement de l'établissement. Ailleurs, on laisse la liberté aux enseignants d'en parler discrètement en classe. La question de l'assistance aux obsèques est souvent la 
pierre de touche de la situation. Ici on n'en parle pas, mais on ne va pas sanctionner l'absence des élèves qui ont voulu s'y rendre. Ailleurs des informations seront officiellement données afin que les élèves puissent s'y rendre s'ils le souhaitent. À l'extrême, dans certaines situations, il a été discuté de la fermeture de l'établissement le jour des funérailles. Cette dernière position paraît actuellement excessive au plus grand nombre. En dehors des questions de l'annonce de l'événement et de la participation aux funérailles, des difficultés de comportement et d'apprentissage peuvent se faire jour après un tel événement ; les responsables de l'établissement ont souvent tendance à faire appel à des ressources extérieures spécialisées, en particulier à des psychologues.

\section{L'AIDE AUX JEUNES ENDEUILLÉS}

Notre association Vivre son Deuil a été amenée, il y a quelques années, à intervenir dans un collège de la région parisienne. Dans une classe de troisième comprenant une trentaine de jeunes d'environ 15 ans, trois étudiants avaient perdu un proche durant le seul mois de décembre : deux étudiants avaient perdu leur mère et le troisième avait perdu une sœur. À la rentrée après les vacances de Noël, l'atmosphère était très lourde, triste et explosive dans la classe qui n'arrivait plus à retrouver son rythme de travail. La directrice, les responsables et les enseignants avaient parlé avec l'ensemble des élèves en classe et avec chacun des adolescents en deuil en particulier, et ils avaient même proposé un lieu et un temps d'écoute pour tous ceux et celles qui souhaitaient rencontrer un adulte pour en parler. Mais rien ne semblait y faire. La directrice, qui avait assisté l'année précédente à notre congrès intitulé "L'enfant face à la mort ", eut l'idée de prendre contact avec nous pour demander conseil. Le président la reçut pendant les vacances d'hiver et, ayant apprécié les interventions entreprises et ne voyant guère quoi ajouter, lui fit part que Jean Monbourquette ${ }^{1}$ a mis en place au Québec au sein de certains établissements, voilà déjà plusieurs années, des groupes de parole pour les enfants en deuil et les enfants en difficulté, en particulier après la séparation des parents. Elle trouva cette information excellente, et à l'étonnement du président qui pressentait toutes les difficultés et les obstacles à une telle réalisation en France, elle dit que c'était ce qu'elle allait faire. Et elle le fit.

Elle obtint successivement le consentement du rectorat, de l'inspection académique, des parents d'élèves et des enseignants de son établissement. Et elle nous demanda de venir animer ce groupe qui débuta quelques semaines plus tard. Ce groupe comprenait six élèves de la classe : les trois endeuillés et trois jeunes dont les parents venaient de divorcer (ce qui est la pratique de Jean Monbourquette). Le groupe s'est réuni quatre fois avant les vacances d'été. Il était animé par deux psychologues de l'association.

Ce groupe eut beaucoup de succès et d'autres élèves demandèrent à y participer ; les enseignants firent également la demande d'un groupe à leur intention, ce qui finalement ne put se réaliser. Mais pour d'autres enfants en difficulté, le groupe fonctionna encore les deux années scolaires suivantes et il aurait sans doute continué si la directrice n'avait pas été déplacée. Les règles d'abstinence (absence de contact des responsables avec les jeunes en dehors du groupe), de libre association et de confidentialité furent respectées. Lors de la première réunion, les six participants ont parlé simplement du décès de leur proche ou du divorce de leurs parents avec plus ou moins d'émotion. Les thèmes des séances suivantes furent libres. Le dessin du sociogénogramme (arbre généalogique comportant les noms des membres de la famille, leurs dates de naissance et de décès et la cause de celui-ci) eut beaucoup de succès et donna lieu à de nombreux échanges. Le désinvestissement scolaire a été abordé et mis en lien avec les souffrances éprouvées par ces adolescents, souffrances ainsi exprimées et reconnues. L'expression de leurs émotions, de leurs préoccupations et de leurs craintes les a soulagés et a atténué les tensions dans le groupe tandis que les résultats du travail scolaire commençaient à s'amender.

D'une manière générale, la famille elle-même en deuil n'est pas souvent en état d'entendre la souffrance de l'enfant. Par ailleurs celui-ci, et encore plus s'il est adolescent, a beaucoup de mal à accepter une aide extérieure, en particulier psychologique ( Je ne suis pas malade »). Aussi, cette démarche essentielle pour la bonne évolution du deuil, épreuve particulièrement difficile durant l'enfance, et encore plus durant l'adolescence, confirme l'importance de l'école en tant que lieu de vie et d'interactions où les élèves qui y passent beaucoup de temps peuvent trouver à s'exprimer librement.

Cette initiative est très intéressante et il est souhaitable qu'elle se répande, mais elle a contre elle d'être très nova- trice : l'institution scolaire française n'est pas ouverte à ce qui ne fait partie des programmes. J'ai oublié de préciser que ces groupes se réunissaient durant le temps scolaire. D'autre part, il ne faut pas se cacher que cette initiative a pu réussir grâce à un heureux concours de circonstances : l'intérêt, la préoccupation, la décision de la directrice, et l'assentiment de toutes les autres instances. Si des parents d'élèves ou des enseignants s'étaient opposés à cette démarche, la considérant étrange, bizarre et inadaptée, elle n'aurait tout simplement pas vu le jour. À l'heure actuelle où nous travaillons activement à la mise en place de programmes d'intervention en cas de mort, de suicide, de catastrophes dans les établissements, il faut bien reconnaître que la mise en place de tels groupes de deuil n'apparaît pas encore comme une priorité.

\section{LE RENOUVEAU D'INTÉRÊT POUR LA MORT ET LE DEUIL}

En ce tournant de siècle et de millénaire, la société moderne vit de grands changements pour tout ce qui concerne la mort et le deuil. La mort est devenue un sujet tabou, écarté du discours social prédominant. De plus, la mort a été prise en charge par les professionnels de la santé. Le deuil, pour sa part est tabou parce qu'il concerne la souffrance. Cependant, comme Louis-Vincent Thomas l'avait très finement indiqué quelques semaines avant son décès, nous assistons à un renouveau d'intérêt social pour la mort et le deuil.

L'extension de la pandémie sidéenne, le développement des soins palliatifs et sans doute les préoccupations éthiques concernant la procréation, le génome, le clonage en sont certainement responsables, au moins en très grande partie. Les manifestations les plus évidentes en sont, parmi d'autres, la multiplication des colloques, des congrès et des conférences tenus des deux côtés de l'Atlantique sur ces thèmes, l'intérêt des médias, la multiplication des publications. Linstitution scolaire ne reste pas à l'écart de ces changements et réalise peu à peu qu'elle a un rôle à jouer dans ces circonstances particulières et inévitables de la vie. Le premier pas de la prise de conscience collective est justement de se rendre compte qu'un lieu de vie qui regroupe plusieurs centaines de personnes est inévitablement confronté un jour ou l'autre à de telles situations et dans des circonstances très différentes.

Cette prise de conscience est évidemment décalée entre les personnels médico-sociaux, les médecins, les infirmières, 
les assistantes sociales, les conseillères d'orientation psychologues (COP) et les responsables administratifs ; les enseignants se situant selon leurs affinités entre ces deux pôles. Ce sont les premiers qui ont réalisé le plus tôt que ces situations nécessitaient une prise en charge spécifique et institutionnelle, et qui ont demandé des formations particulières sur ces sujets. C'est ainsi que l'association Vivre son Deuil et la Société de Thanatologie, et plus la première que la seconde, ont été sollicitées pour mettre en place et assurer de telles formations tant au niveau d'un établissement que, maintenant davantage, au niveau des Académies. Nos relations de réseaux avec les antennes régionales de Vivre son Deuil, en particulier Vivre son Deuil Nord Pas-de-Calais et Vivre son Deuil Picardie, mais aussi avec d'autres associations comme les différents JALMALV (Jusqu'à la mort accompagner la vie) nous ont fait savoir que de telles formations s'étaient mises en place dans d'autres régions, certaines arrivant même à toucher les chefs d'établissements ${ }^{2}$. Deux Instituts Universitaires de Formation des Maîtres (IUFM) nous ont demandé d'intervenir au cours de cette année universitaire sur ces sujets auprès de leurs étudiants. Ceci correspond bien à une évolution générale puisque l'enseignement des soins palliatifs avec un module sur le deuil et un autre sur les rites est entré dans les études médicales à partir de l'an dernier et est devenu obligatoire pour les personnels funéraires à partir de 1993.

\section{FORMER DANS LE CONTEXTE SCOLAIRE}

Le but des responsables médicosociaux du rectorat qui ont initié des formations sur la mort et le deuil à l'intention de leurs personnels est double : sensibiliser et former les intervenants naturels de proximité et mettre en place un protocole d'intervention pour l'institution.

De fait, ces cycles de formation de plusieurs jours commencent par l'acquisition de connaissances fondamentales sur les idées des enfants et des adolescents au sujet de la mort, sur le déroulement et le vécu du deuil chez les uns et les autres, sur les particularités de leur travail de deuil, sur les risques de complications, sur les issues ultérieures de tels traumatismes durant la jeunesse et sur l'accompagnement du deuil dans ces populations. De tels enseignements sont naturellement interactifs puisqu'ils sont dispensés à des professionnels de terrain qui ont déjà de l'expérience et ont ren- contré des situations de ce type. Il est aussi très important, au cours de ces formations, que soient ménagés des moments de partage sur les situations difficiles qui ont été vécues dans le passé et dont les participants ont conservé un souvenir mitigé. C'est l'occasion à la fois d'exprimer le trop-plein d'émotions bloquées à l'intérieur et de réaliser ce qui aurait pu être fait de manière plus adéquate, l'avis des autres membres du groupe étant alors essentiel.

Tout, dans ces domaines, n'est pas affaire de connaissance, mais des connaissances précises sont indispensables ; lorsqu'elles ont été acquises, chacun se sent plus à l'aise pour donner son opinion et intervenir sur le terrain. Il est bien difficile de comprendre les enfants en deuil si on ignore que pour plusieurs d'entre eux la mort n'est pas naturelle ("On ne meurt pas ; on est tué »), qu'elle est réversible et qu'elle est contagieuse, si on ne sait pas que, à côté de ces conceptions irréalistes et purement subjectives que certains enfants conservent jusqu'à la fin de l'adolescence, ils acquièrent également des connaissances objectives sur la mort dans un ordre déterminé selon leur évolution psychologique.

La mort, pour les tout-petits, représente d'abord l'absence. Elle est ensuite la perte de toutes les fonctions vitales. Puis, vers quatre ans, ils comprennent qu'elle est irréversible, ils mettront toutefois des années à l'accepter. Après cet âge, la mort est comprise comme universelle. Un peu plus tard, elle apparaît comme une qualité fondamentale et inaliénable de la vie. L'important est aussi que ces conceptions subjectives et ces connaissances objectives ne se contredisent pas mutuellement et continuent longtemps de cheminer côte à côte ; l'attitude de l'enfant étant tantôt sous l'obédience des premières et tantôt en relation avec les secondes. Il passe des unes aux autres avec une liberté qui nous déconcerte. Il n'a pas la même conception de la réalité que nous. Pour lui, on peut être vivant et mort à la fois. Des enfants en deuil savent bien que leur papa est mort, mais ils ne peuvent s'empêcher d'en attendre le retour souvent en lui parlant, parfois en lui écrivant. La survivance de ce "parent imaginaire " ne doit en aucun cas être considérée comme une complication ; c'est un élément fondamental du deuil des enfants.

Le deuil chez les enfants et les adolescents a aussi des particularités. Les enfants surtout ont davantage de mal à extérioriser leur chagrin s'ils n'y sont pas encouragés par les adultes. La dépression de deuil des enfants n'est pas identique à celle des adultes. Chez eux, elle se manifeste par des modifications du caractère, de l'humeur, de comportement, conduit souvent à des prises de risque, au retour de l'énurésie et enfin à la survenue d'une authentique maladie organique, ce qui attire toujours l'attention des pédiatres sur l'existence éventuelle de facteurs psychosociaux dans la vie de l'enfant malade. Il est encore nécessaire de savoir que le deuil chez les enfants ne va jamais jusqu'à son terme ; demeure toujours une part de souffrance qui réapparaîtra au cours d'épreuves ultérieures dans la suite de la vie. C'est la raison pour laquelle il est si difficile d'apprécier avec justesse le pronostic de ces deuils dans l'enfance ; nous risquons tout autant d'en sous-évaluer l'importance comme de la surévaluer.

Pour ce qui est du travail de deuil de l'enfant, l'attention doit être particulièrement portée sur le fait que, pour accepter la réalité de la mort, l'enfant a besoin de participer aux derniers moments, de dire au revoir au défunt et d'être acteur dans les funérailles, ce qui est aux antipodes de la tendance sociologique dominante à l'heure actuelle qui est encore d'écarter les enfants des abords de la mort. Or, cette tendance est en train d'évoluer comme le montrent les derniers sondages et enquêtes. Un autre point capital à considérer concerne le fait que l'enfant se sent toujours coupable de la mort des êtres qui l'entourent en raison des sentiments de toute-puissance psychique qui l'habitent, des restes de sa pensée magique, de son ambivalence. Il est donc essentiel de le rassurer sur ce point.

Lorsque la population est constituée de pré-adolescents et d'adolescents, c'est-à-dire à partir, chez nous, du collège, des formations de ce type comprennent toujours un temps de réflexion sur le suicide. Outre les données épidémiologiques et sociologiques, nous nous attachons toujours à faire réfléchir sur le caractère unique de chaque situation et sur les différents aspects psychologiques, les différentes motivations. Bien sûr le suicide est envisagé comme un agir, un acte de violence pour celui qui le fait comme pour son entourage, agir qui signe à la fois la faillite de la communication et le summum du désespoir. Il est aussi considéré comme un mystère quels que soient les mots laissés par le suicidé par écrit ou sur son ordinateur, comme un message aussi et parfois avec une dimension de sacrifice. L'attention est particulièrement portée sur les particu- 
larités du deuil après le suicide qui est un deuil traumatique laissant derrière lui des images fortes et obsédantes et une dose de culpabilité considérable. Enfin, il est souvent utile et souvent demandé de compléter ce cycle de formation par une réflexion sur les préoccupations des adolescents vis-à-vis de la mort.

\section{PROTOCOLE D'INTERVENTION DANS L'ÉCOLE EN DEUIL}

Nous pouvons alors en arriver à la discussion du protocole d'intervention réalisé de manière progressive et conviviale avant d'être couché par écrit pour être soumis aux différents participants. Après quoi il sera diffusé au sein des établissements selon un plan établi en commun.

Les grandes lignes de ce protocole sont actuellement les suivantes, le présupposé fondamental étant que l'établissement doit, en cas de décès d'un élève ou d'un adulte de l'établissement, réagir institutionnellement, officiellement. À distance, en amont des événements, une cellule de crise est constituée qui comprend : l'équipe de direction, l'équipe éducative et l'équipe médico-sociale. Lorsqu'arrive un événement de ce genre, la présence du chef d'établissement est indispensable à la mise en place des actions à mener. En premier lieu, il vérifie la nouvelle auprès de la famille ou, le cas échéant, auprès de la gendarmerie ou de l'hôpital. Puis, il convoque dans les plus brefs délais la réunion de la cellule de crise afin de recenser les personnes concernées par ce décès, afin aussi d'organiser et de planifier les actions à mener. La cellule de crise veille à ce que l'annonce officielle soit faite à toutes les personnes de l'établissement, soit directement par un document ou une lettre rédigée par ses membres, soit indirectement par affichage du contenu de cette lettre qui doit atteindre tous les adultes de l'établissement en veillant aux absents (congés ou maladie).

Les élèves sont informés par les enseignants dès la première heure de cours, en donnant connaissance du contenu du document à toutes les classes concernées. Un membre de la direction annonce officiellement la mort, la date, le lieu, éventuellement les circonstances (accident, maladie), adresse un mot de sympathie et de regret à l'égard du défunt, exprime le chagrin collectif, transmet des informations sur les funérailles et leur déroulement pratique, transmet également des informations sur les possibilités d'aide (personnes ressources, lieux et heures) et de manifestations de sympathie : minute de silence, collecte (fleurs, plaque), cartes de condoléances, annonce d'une réunion générale de toutes les personnes de l'établissement qui le souhaiteront le lendemain ou le surlendemain afin d'apporter de nouvelles informations.

À l'arrivée des élèves, le rôle des enseignants est de faire l'annonce en leur laissant le temps de réagir, leur proposer d'en parler collectivement ou individuellement, autoriser les élèves à quitter la classe, accompagnés, pour se rendre vers une personne ressource de l'établissement, être vigilant aux différentes réactions, l'absence de manifestations devant être prise en compte, annoncer la réunion générale. L'enseignant informera ensuite la cellule de crise des réactions dans sa classe. L'activité scolaire reprendra son cours, mais certains examens et travaux pourront être reportés. En aucun cas les cours ne seront suspendus.

Les personnels médico-sociaux et les conseillers d'orientation psychologiques de l'établissement assurent le suivi, le soutien et l'encadrement adaptés aux besoins spécifiques de chacun, adultes et élèves ; ils recevront et accueilleront les personnes qui le souhaitent, repéreront les élèves et les adultes les plus en souffrance pour les aider à verbaliser, à exprimer ce qu'elles ressentent et les orienter éventuellement vers des ressources extérieures. Ils alerteront les familles des jeunes les plus en difficulté.

Les membres de la cellule de crise feront le point de la journée, décideront éventuellement de faire appel à des ressources extérieures à l'établissement et prépareront la réunion générale.

Dans les jours qui suivent, seront mis en place l'équipe ressource et la réunion générale. Celle-ci a été annoncée dès le début. Elle a lieu à la fin des cours le deuxième ou le troisième jour. Toutes les personnes de l'établissement y sont conviées et y viennent celles qui le souhaitent. Elles sont accueillies de préférence en musique (douce, calme, recueillie). Le directeur dit d'emblée que la réunion a lieu pour parler de celui ou celle qui est mort et demande un moment de silence. Il fournit ensuite plus d'informations (dans la mesure du possible) sur les circonstances du décès, dit un mot sympathique et chaleureux sur la personne qui est morte et dont on porte collectivement témoignage, il fait état de quelques réactions de chagrin et de sympathie qui lui ont été rapportées, propose la parole à ceux qui désirent dire un mot. Un autre responsable parle alors des funérailles et un troisième enfin reprécise les possibilités d'aide et de soutien.
À plus long terme, s'il s'agit d'une mort par accident ou par suicide, une conférence-débat sur les conduites à risque de l'adolescence sera utilement mise en place dans les mois suivants en faisant appel à un intervenant extérieur. Reste à rappeler le souvenir et à discuter d'éventuelles commémorations à distance, sachant que les parents, proches et amis y sont très sensibles. Les enseignants sont encouragés à parler de l'élève qui est mort un ou deux mois plus tard. À l'anniversaire de la mort d'une personne de l'établissement, la cellule de crise peut décider d'apposer des affichettes pendant quelques jours pour en raviver le souvenir.

De toutes les façons, il reste encore à se tenir très attentif à tous les signes de malaise de toutes les personnes les plus fragiles de l'établissement et ne pas hésiter à leur proposer de l'aide par tout moyen qui paraîtra le plus adapté.

\section{CONCLUSION}

Cette prise de conscience, cette réflexion, ces formations, l'établissement d'un protocole et la mise en place d'une cellule de crise ne sont encore le fait que de quelques Académies, mais il n'est pas déraisonnable de penser que ces procédures soient de mieux en mieux reçues et acceptées par les milieux scolaires. On voit déjà que le plus grand nombre d'établissements atteints par une catastrophe, et maintenant par un suicide, demandent l'aide de professionnels extérieurs, pas nécessairement bien formés à ce type particulier d'intervention. Il est plus que vraisemblable que les échanges vont se multiplier.

Mais il reste encore à réfléchir sur la faisabilité de ces pratiques, aux résultats de leur confrontation avec les situations concrètes qui sont toujours singulières et qui, du fait de leur brutalité et de leur caractère inhabituel, risquent toujours de nous prendre en défaut. C'est la raison pour laquelle les équipes au travail ont décidé de se retrouver d'année en année.

\section{Notes}

1 Jean MONBOURQUETTE, Rassembler groupe d'entraide pour personnes en deuil : comment l'organiser et le diriger, Ottawa, Novalis, 1993, 72 pages ; "Les groupes d'entraide pour adolescents endeuillés ", Frontières, vol. 9, no 3, hiver 1997, p. 23-27.

2 Association Vivre son Deuil http://www.vivresondeuil.asso.fr/ ; Association JALMALV

http://www.clparc-beauvais.fr 